\title{
AN AUTOMATIC SNOW DEPTH METER BY AN INFRARED TECHNIQUE
}

\author{
By TAdashi Kimura
}

(Institute of Snow and Ice Studies, National Research Center for Disaster Prevention, Suyoshi, Nagaoka-shi, Niigata-ken, Japan)

Abstract. An automatic snow-depth meter was constructed using an infrared beam from a gallium-arsenide photo-emission diode and an infrared detector consisting of a silicon photocell. As the field of view of both photoelectric elements is $30^{\circ}$, high mechanical accuracy is not required. The equipment consists of two poles of the same size, $4.8 \mathrm{~m}$ high and $20 \mathrm{~cm}$ by $20 \mathrm{~cm}$ wide, an operation controller, and a digital recorder. The two poles were set up on the ground facing each other at a distance of $5 \mathrm{~m}$. One pole has the photo-emission diode and the other the infrared detector. In each pole, a long slit $4 \mathrm{~cm}$ wide and $4 \mathrm{~m}$ long was installed. Through one slit the infrared beam comes out and is received through the other slit. To avoid catching snow or rain in the slits, air was ejected through them.

The meter measures the snow depth at pre-set time intervals of more than ten minutes. Both the emitter and the detector rest at the top of the poles between the time intervals, and then move down as far as the snow surface level and then return to the top, keeping the same level as each other all the time. The detector recognizes the snow surface level when the received infrared beam comes down to $50 \%$ of the emitted intensity. At present, sudden noises such as occurs if the sun shines, are eliminated by an electronic pulse technique and an optical filter.

The meter has operated successfully in two seasons (1972-74) in mean air temperatures of o to $-5{ }^{\circ} \mathrm{C}$ without any trouble, although the whole apparatus has been exposed outdoors throughout the whole seasons. The following results were obtained:

(I) With any condition of snow surface, the accuracy of the measurements was within $\pm \mathrm{r} 0 \mathrm{~mm}$ for snow depth compared with that measured by a snow stick and was $\pm 2 \mathrm{~mm}$ compared with a concrete block.

(2) The system of ejecting air through the slits worked quite well, not only keeping the slits open during the period of measurements but preventing moisture inside the poles.

(3) The percentage of inevitable mistakes of the meter, mainly caused by a heavy snowstorm or in other words by a high snow density in the atmosphere, was $0.5 \%$ of all readings at Nagaoka, Japan. 